\section{CT Peritoneal Cancer Index in Colorectal Peritoneal Carcinomatosis}

\section{TO THE EDITORS:}

Thank you for inviting me to respond to the comments made by Drs. Esquivel and Chua regarding our study on CT peritoneal Cancer Index (PCI) in colorectal peritoneal carcinomatosis (CRPC). ${ }^{1}$ The question about how CT-PCI contributed to the selection of our 19 patients seems to be invalid, because our study is an observational analysis of these 19 patients who had already undergone peritonectomy. Obviously, CT-PCI did not influence our decision to proceed with the surgery at the time. However, I would like to emphasize two points that we have learnt from our experience. First, it was to our surprise that the mean operative PCI nearly doubled that approximated by CT $(P=0.001)$. Numerous studies have demonstrated a survival benefit with a low PCI score, although the absolute cutoff point is not established. I think it is reasonable to suggest that at our institution, patients with a preoperative CT-PCI $>15$ should not be considered for the combined treatment in CRPC, because the intraoperative PCI will be much higher. Second, to really find out how CT-PCI contributes to patient selection, we must collect the data prospectively for all patients referred for peritonectomy, i.e., not just those who had undergone the surgery but also the ones who had an open-close procedure due to high intraoperative PCI and the ones who had been excluded preoperatively due to high CT-PCI. The outcomes must be recorded on an intention-to-treat basis. Many centers, including ours, report outcomes only for patients who received peritonectomy. Although this may be predictive of outcome, it is not useful in terms of patient selection. The prospective data registry on CRPC will provide us with meaningful data, but it also lacks treatment outcomes regarding patients who have been excluded from peritonectomy. $^{2}$

\section{Tristan D. Yan, BSc(Med) MBBS PhD}

Department of Surgery, St. George Hospital, University of New South Wales, Sydney, NSW, Australia

e-mail: tristan.yan@unsw.edu.au

Published Online: 9 July 2009

(C) Society of Surgical Oncology 2009

\section{REFERENCES}

1. Koh JL, Yan TD, Glenn D, Morris DL. Evaluation of preoperative computed tomography in estimating peritoneal cancer index in colorectal peritoneal carcinomatosis. Ann Surg Oncol. 2009;16:327-33.

2. Esquivel J, Sticca R, Sugarbaker P, et al. Cytoreductive surgery and hyperthermic intraperitoneal chemotherapy in the management of peritoneal surface malignancies of colonic origin: a consensus statement. Ann Surg Oncol. 2007;14:128-33. 